\title{
Solving parametrized systems of Pell equations
}

\author{
by \\ Paulo Ribenboim (Kingston) \\ To my long time friend Pierre Samuel, \\ octogenarian in love with Diophantus
}

1. Introduction. Let $F>1$ be a square-free integer. In his papers [3]-[6] Ljunggren studied the quartic equation

$$
x^{4}-F y^{2}=1
$$

(and similar equations) and proved that the equation has at most two solutions $(x, y)$ in positive integers. He also gave an algorithm to find the solutions.

When $F=p$ is a prime number, Ljunggren showed that

$$
x^{4}-p y^{2}=1
$$

has no solution in positive integers when $p \neq 5,29$. Moreover if $p=5$ the only solution is $(3,4)$ and if $p=29$ the only solution is $(99,1820)$. In [12] Samuel gave another proof for $p=5$.

The proof of this result leads to the study of the systems

$$
\left\{\begin{array}{l}
x^{2}-2 y^{2}= \pm 1 \\
x^{2}-2 p y^{2}=\mp 1
\end{array}\right.
$$

In our paper we shall need binary recurring sequences. Let $P>0, Q \neq 0$ be integers such that $D=P^{2}-4 Q>0$. We shall consider the Lucas sequences $\left(U_{n}\right)_{n},\left(V_{n}\right)_{n}$ with parameters $(P, Q)$ :

$$
\begin{array}{ll}
U_{0}=0, \quad U_{1}=1, & V_{0}=2, \quad V_{1}=P, \\
U_{n}=P U_{n-1}-Q U_{n-2} \text { for } n \geq 2, & V_{n}=P V_{n-1}-Q V_{n-2} \text { for } n \geq 2, \\
U_{n}=\left(-1 / Q^{n}\right) U_{-n} \text { for } n<0 ; & V_{n}=\left(1 / Q^{n}\right) V_{-n} \text { for } n<0 .
\end{array}
$$

2000 Mathematics Subject Classification: Primary 11D09. 
As is easily seen, the above recurrences still hold for any integer $n$. When needed, we shall use the notation $U_{n}=U_{n}(P, Q), V_{n}=V_{n}(P, Q)$.

Given the square-free integer $F>1$, let $\varepsilon=c+d \sqrt{F}$ be the fundamental unit of the $\operatorname{ring} \mathbb{Z}[\sqrt{F}]$, so $c>0, d>0$. As is well known, $c$ and $d$ are effectively bounded in terms of $F$. Let $P=2 c, Q=c^{2}-D d^{2}= \pm 1$, let $U_{n}=U_{n}(2 c, Q), V_{n}=V_{n}(2 c, Q)$. Then

$$
\varepsilon^{n}=\frac{V_{n}}{2}+d U_{n} \sqrt{F} .
$$

As is easily seen, $V_{n}$ is even for every $n \in \mathbb{Z}_{-}$and $V_{2 n} \equiv 2(\bmod 4)$ for every $n \in \mathbb{Z}$. If $s \geq 1$ we define

$$
k_{s}=\frac{1}{4}\left(2+Q^{s} V_{2 s}\right), \quad h_{s}=\frac{1}{4}\left(2-Q^{s} V_{2 s}\right),
$$

so $h_{s}, k_{s} \neq 0,1$ and $k_{s}+h_{s}=1$.

(1.1) Theorem. Let $F>1, G \geq 1$ be square-free integers, let $s \geq 1$, $f \neq 0$ and $g=f k_{s}$ or $g=f h_{s}$. Then there exists an integer $N>0$, effectively computable in terms of $F, G, f$ and $s$, such that if $p=1$ or $p$ is a prime number, if $x \geq 0, y \geq 0, z>0$ are integers such that

$$
\left\{\begin{array}{l}
x^{2}-F y^{2}=f \\
x^{2}-p G z^{2}=g
\end{array}\right.
$$

then $x, y, z, p<N$.

\section{Preliminaries}

A. Binary recurring sequences. Let $P>0, Q \neq 0$ with $D=P^{2}-4 Q>0$. We gather some properties of $U_{n}=U_{n}(P, Q)$ and $V_{n}=V_{n}(P, Q)$ which will be needed in this paper.

Let $\alpha, \beta$ be the roots of $X^{2}-P X+Q$, so

$$
\begin{gathered}
\alpha=\frac{P+\sqrt{D}}{2}, \quad \beta=\frac{P-\sqrt{D}}{2}, \\
\alpha+\beta=P, \quad \alpha \beta=Q, \quad \alpha-\beta=\sqrt{D} .
\end{gathered}
$$

For each $n \in \mathbb{Z}$ :

$$
U_{n}=\frac{\alpha^{n}-\beta^{n}}{\alpha-\beta}, \quad V_{n}=\alpha^{n}+\beta^{n} .
$$

If $m, n \in \mathbb{Z}$ :

$$
\begin{gathered}
2 U_{m+n}=U_{m} V_{n}+U_{n} V_{m} \\
U_{m+n}=U_{m} V_{n}-Q^{n} U_{m-n}, \quad V_{m+n}=V_{m} V_{n}-Q^{n} V_{m-n} \\
U_{2 m}=U_{m} V_{m}, \quad V_{2 m}=V_{m}^{2}-2 Q^{m} \\
V_{m}^{2}-D U_{m}^{2}=4 Q^{m}
\end{gathered}
$$


The following lemma will be required:

(2.1) Lemma. For every $s \geq 1$ and $n \in \mathbb{Z}$ we have

(a) $V_{n}^{2}-V_{n-s} V_{n+s}=Q^{n-s}\left(2 Q^{s}-V_{2 s}\right)$,

(b) $V_{n}^{2}-D U_{n-s} U_{n+s}=Q^{n-s}\left(2 Q^{s}+V_{2 s}\right)$,

(c) $D\left(U_{n}^{2}-U_{n-s} U_{n+s}\right)=Q^{n-s}\left(-2 Q^{s}+V_{2 s}\right)$,

(d) $D U_{n}^{2}-V_{n-s} V_{n+s}=-Q^{n-s}\left(2 Q^{s}+V_{2 s}\right)$.

Proof. We just prove (a):

$$
\begin{aligned}
V_{n}^{2}-V_{n-s} V_{n+s}= & \left(\alpha^{n}+\beta^{n}\right)^{2}-\left(\alpha^{n-s}+\beta^{n-s}\right)\left(\alpha^{n+s}+\beta^{n+s}\right) \\
= & \alpha^{2 n}+\beta^{2 n}+2(\alpha \beta)^{n} \\
& -\left[\alpha^{2 n}+\beta^{2 n}-(\alpha \beta)^{n-s}\left(\alpha^{2 s}+\beta^{2 s}\right)\right] \\
= & Q^{n-s}\left(2 Q^{s}-V_{2 s}\right) .
\end{aligned}
$$

Now we assume $\operatorname{gcd}(P, Q)=1$. If $m, n \geq 1$ and $d=\operatorname{gcd}(m, n)$ then

$$
\begin{aligned}
& \operatorname{gcd}\left(U_{m}, U_{n}\right)=U_{d}, \\
& \operatorname{gcd}\left(V_{m}, V_{n}\right)= \begin{cases}V_{d} & \text { if } m / d \text { and } n / d \text { are odd, } \\
1 \text { or } 2 & \text { otherwise, }\end{cases} \\
& \operatorname{gcd}\left(U_{m}, V_{n}\right)= \begin{cases}V_{d} & \text { if } m / d \text { is even, } \\
1 \text { or } 2 & \text { otherwise }\end{cases} \\
& \operatorname{gcd}\left(U_{n}, Q\right)=1, \quad \operatorname{gcd}\left(V_{n}, Q\right)=1 .
\end{aligned}
$$

If $a, b \in \mathbb{Z}$, not both equal to 0 , let

$$
W_{n}=a U_{n}+b V_{n} \quad \text { for all } n \in \mathbb{Z} .
$$

Then

$$
W_{n}=P W_{n-1}-Q W_{n-2} \quad \text { for all } n \in \mathbb{Z} \text {. }
$$

We also have

$$
W_{m+n}=W_{m} V_{n}-Q^{n} W_{m-n} \quad \text { for all } m, n \in \mathbb{Z} .
$$

If $\operatorname{gcd}(P, Q)=1$ then

$$
\operatorname{gcd}\left(W_{n}, Q\right)=\operatorname{gcd}\left(W_{1}, Q\right) \quad \text { for all } n \geq 1 .
$$

The following lemma will also be required:

(2.2) Lemma. Assume that $W_{n} \neq 0$ for all $n \geq 1$ and $\operatorname{gcd}\left(W_{1}, Q\right)=1$. If $t \geq 1$ then $\operatorname{gcd}\left(W_{n}, W_{n+t}\right)$ divides $W_{1} W_{2} \cdots W_{t}$ for all $n \geq 1$.

Proof. The lemma is trivial for $n=1, \ldots, t$. Let $t \leq n$ and assume the lemma true for $1,2, \ldots, n$. Let $d=\operatorname{gcd}\left(W_{n+1}, W_{n+1+t}\right)$. We have $W_{n+1+t}=$ $W_{n+1} V_{t}-Q^{t} W_{n+1-t}$. Since $1 \leq n+1-t \leq n$ and $\operatorname{gcd}\left(W_{n+1}, Q\right)=$ $\operatorname{gcd}\left(W_{1}, Q\right)=1$, it follows that $d$ divides $\operatorname{gcd}\left(W_{n+1-t}, W_{n+1}\right)$, which, by induction, divides $W_{1} W_{2} \cdots W_{t}$, thus concluding the proof. 
We shall need the following theorem (see Shorey \& Tijdeman [14], Shorey $\&$ Stewart [13], Pethő [9]), which we quote in the special case needed in this paper.

With the preceding notations:

(2.3) Theorem. Assume that $\operatorname{gcd}(P, Q)=1$ and $D \neq 0$. Let $a, b \in \mathbb{Z}$, not both equal to 0 , let $W_{n}=a U_{n}+b V_{n}$ for all $n \in \mathbb{Z}$. Let $A>0$ be a square-free integer. Then there exists $N>0$, effectively computable in terms of $P, Q, a, b, A$ such that if $n \geq 0$ and $W_{n}=A \square$ (where $\square$ denotes a non-zero integer which is a square) then $n<N$.

The proof of this theorem involves inequalities of Baker for linear forms in logarithms and the constant $N$ provided by the proof is usually very large.

For special sequences, the explicit determination of squares and doublesquares has been achieved. We quote a few results for sequences with parameters $P$ even and $Q= \pm 1$.

For $P=2, Q=1, U_{n}$ and $V_{n}$ are the Pell numbers and we have:

$$
\begin{array}{llrl}
\left\{n \mid U_{n}=\square\right\} & =\{1,7,-1,-7\}, & & \left\{n \mid U_{n}=2 \square\right\}=\{2\}, \\
\left\{n \mid V_{n}=\square\right\}=\emptyset, & & \left\{n \mid V_{n}=2 \square\right\}=\{0,1\} .
\end{array}
$$

The above results are due to Ljunggren [5]; the determination of the square Pell numbers required deep arguments.

Ljunggren [5] and Cohn [1] studied the sequences of numbers $U_{n}(4,-1)$ and $V_{n}(4,-1)$ :

$$
\begin{array}{ll}
\left\{n \mid U_{n}(4,-1)=\square\right\}=\{1,2,-1\}, & \left\{n \mid U_{n}(4,-1)=2 \square\right\}=\{4\}, \\
\left\{n \mid V_{n}(4,-1)=\square\right\}=\{1\}, & \left\{n \mid V_{n}(4,-1)=2 \square\right\}=\{0,2,-2\} .
\end{array}
$$

In [1] Cohn obtained more results about squares and double squares in the sequences $U_{n}(2 c, \pm 1)$ and $V_{n}(2 c, \pm 1)$, for special values of $2 c$.

The reader may obtain more information about recurring sequences in Ribenboim [11] (see Chapter 1 entitled "The Fibonacci Numbers and the Arctic Ocean"). More specifically about Pell numbers, see Ribenboim [10].

B. Pell equations. We keep the same notations: $F>1, f \neq 0, \varepsilon=$ $c+d \sqrt{F}$ is the fundamental unit of $\mathbb{Z}[\sqrt{F}]$, so $c \geq 1, d \geq 1 ; P=2 c$, $Q=c^{2}-F d^{2}= \pm 1, U_{n}=U_{n}(2 c, Q), V_{n}=V_{n}(2 c, Q)$. We consider solutions of $x^{2}-F y^{2}=f$.

Two solutions $(x, y)$ and $\left(x^{\prime}, y^{\prime}\right)$ of the Pell equation are said to be equivalent if there exists $n \in \mathbb{Z}$ such that

$$
Q^{n}=1 \quad \text { and } \quad \frac{x+y \sqrt{F}}{x^{\prime}+y^{\prime} \sqrt{F}}=\varepsilon^{n} .
$$

If $Q=1$ let $c^{\prime}+d^{\prime} \sqrt{F}=c+d \sqrt{F}=\varepsilon$. If $Q=-1$ let

$$
c^{\prime}+d^{\prime} \sqrt{F}=\left(c^{2}+d^{2} F\right)+2 c d \sqrt{F}=\varepsilon^{2} .
$$


We note that if $c=1$ then $Q=-1$ so $c^{\prime}>1$.

A solution $(a, b)$ with $a \geq 0$ and $b \geq 0$ is called a fundamental solution if the following inequalities are satisfied:

$$
0 \leq a \leq \sqrt{\frac{\left(c^{\prime}+\delta\right)|f|}{2}}, \quad 0 \leq b \leq d^{\prime} \sqrt{\frac{|f|}{2\left(c^{\prime}+\delta\right)}}
$$

where $\delta=|f| / f$.

Nagell proved (see [7] and [8]):

(2.4) Theorem. Every solution $(x, y)$ with $x \geq 0, y \geq 0$ of $x^{2}-F y^{2}=f$ is equivalent to a fundamental solution.

3. Proof of Theorem (1.1). We divide the proof into three parts.

$\left.1^{\circ}\right)$ Let $S$ be the set of all $(x, y, z, p)$ such that $x \geq 0, y \geq 0, z>0, p=1$ or $p$ is a prime number and

$$
\left\{\begin{array}{l}
x^{2}-F y^{2}=f \\
x^{2}-p G z^{2}=f k_{s}
\end{array}\right.
$$

[The proof when $g=f h_{s}$ is similar and will not be given.]

Let $T$ be the set of all $(x, y)$ such that $x \geq 0, y \geq 0, x^{2}-F y^{2}=f$ and there exists $(z, p)$ such that $(x, y, z, p) \in S$. Clearly, it suffices to show that the set $T$ is effectively computable.

By the theorem of Nagell (2.4) if the equation $x^{2}-F y^{2}=f$ has solutions, then it has a non-empty effectively computable set of fundamental solutions and every solution $(x, y)$, with $x \geq 0, y \geq 0$ is given by a relation $x+y \sqrt{F}=$ $(a+b \sqrt{F}) \varepsilon^{n}$, where $a \geq 0, b \geq 0,(a, b)$ is a fundamental solution, $\varepsilon=c+d \sqrt{F}$ is the fundamental unit of $\mathbb{Z}[\sqrt{F}], Q=c^{2}-d^{2} F, Q^{n}=1$.

We fix an arbitrary fundamental solution $(a, b)$ and write

$$
x_{n}+y_{n} \sqrt{F}=(a+b \sqrt{F}) \varepsilon^{n}=(a+b \sqrt{F})\left(\frac{V_{n}}{2}+d U_{n} \sqrt{F}\right),
$$

where $U_{n}=U_{n}(2 c, Q)$ and $V_{n}=V_{n}(2 c, Q)$. So

$$
x_{n}=a \frac{V_{n}}{2}+b d F U_{n}, \quad y_{n}=a d U_{n}+b \frac{V_{n}}{2} .
$$

It suffices to show that the set $R=\left\{n>s \mid\left(x_{n}, y_{n}\right) \in T\right\}$ is effectively bounded.

$2^{\circ}$ ) We show that if $Q^{n}=1$ and $s \geq 1$ then

$$
x_{n}^{2}-F y_{n-s} y_{n+s}=f k_{s} .
$$

Indeed:

$$
x_{n}^{2}=\left(a \frac{V_{n}}{2}+b d F U_{n}\right)^{2}=a^{2} \frac{V_{n}^{2}}{4}+\frac{b^{2} F}{4} D U_{n}^{2}+a b d F U_{2 n} .
$$


Next

$$
\begin{aligned}
F y_{n-s} y_{n+s} & =F\left(b \frac{V_{n-s}}{2}+a d U_{n-s}\right)\left(b \frac{V_{n+s}}{2}+a d U_{n+s}\right) \\
& =\frac{b^{2} F}{4} V_{n-s} V_{n+s}+a b d F U_{2 n}+a^{2} d^{2} F U_{n-s} U_{n+s} \\
& =\frac{b^{2} F}{4} V_{n-s} V_{n+s}+a b d F U_{2 n}+\frac{a^{2}}{4} D U_{n-s} U_{n+s} .
\end{aligned}
$$

In the above calculation we used identities indicated in Section 2. It follows that

$$
x_{n}^{2}-F y_{n-s} y_{n+s}=\frac{a^{2}}{4}\left(V_{n}^{2}-D U_{n-s} U_{n+s}\right)+\frac{b^{2} F}{4}\left(D U_{n}^{2}-V_{n-s} V_{n+s}\right) .
$$

By Lemma (2.1) we have

$$
\begin{aligned}
x_{n}^{2}-F y_{n-s} y_{n+s} & =\frac{a^{2}}{4} Q^{n s}\left(2 Q^{s}+V_{2 s}\right)-\frac{b^{2} F}{4} Q^{n s}\left(2 Q^{s}+V_{2 s}\right) \\
& =\frac{f}{4}\left(2+Q^{s} V_{2 s}\right)=f k_{s},
\end{aligned}
$$

because $Q^{n}=1$. [For the proof of the theorem when $g=f h_{s}$ we need the relation

$$
x_{n}^{2}-x_{n-s} x_{n+s}=f h_{s},
$$

which is established in a similar way.]

$\left.3^{\circ}\right)$ By Lemma $(2.2)$ for every $n>s, \operatorname{gcd}\left(y_{n-s}, y_{n+s}\right)$ divides $y_{1} y_{2} \cdots y_{2 s}$; we note that the integer $y_{1} y_{2} \cdots y_{2}$ is effectively computable in terms of $F$, $s$ and the chosen fundamental solution $(a, b)$. For every positive integer $e$ dividing $y_{1} y_{2} \cdots y_{2 s}$, let

$$
R_{e}=\left\{n \in R \mid \operatorname{gcd}\left(y_{n-s}, y_{n+s}\right)=e\right\} .
$$

Let $n \in R_{e}$, so from $F y_{n-s} y_{n+s}=p G z^{2}$ it follows that

$$
e^{2} F^{2} \frac{y_{n-s}}{e} \cdot \frac{y_{n+s}}{e}=p F G \square=p^{\delta} H \square,
$$

where $\delta=0$ or $1, p \nmid H, H$ is square-free and $H \mid F G$. Hence

$$
\frac{y_{n-s}}{e} \cdot \frac{y_{n+s}}{e}=p^{\delta} H \square .
$$

Let $\mathcal{H}=\left\{\left(H^{\prime}, H^{\prime \prime}\right) \mid H^{\prime} H^{\prime \prime}\right.$ is square-free, $H^{\prime} H^{\prime \prime} \mid F G$ and $\left.\operatorname{gcd}\left(H^{\prime}, H^{\prime \prime}\right)=1\right\}$, so $\mathcal{H}$ is effectively computable. For each $\left(H^{\prime}, H^{\prime \prime}\right)$ let $R_{e,\left(H^{\prime}, H^{\prime \prime}\right)}^{\prime}$ be the set of all $n \in R_{e}$ such that

$$
\frac{y_{n-s}}{e}=p^{\delta} H^{\prime} \square, \quad \frac{y_{n+s}}{e}=H^{\prime \prime} \square .
$$

By Theorem (2.3) the set $\left\{n+s \mid n \in R_{e,\left(H^{\prime}, H^{\prime \prime}\right)}^{\prime}\right\}$, hence also $R_{e,\left(H^{\prime}, H^{\prime \prime}\right)}^{\prime}$ is effectively bounded. Similarly, let $R_{e,\left(H^{\prime}, H^{\prime \prime}\right)}^{\prime \prime}$ be the set of $n \in R_{e}$ such 
that

$$
\frac{y_{n-s}}{e}=H^{\prime} \square, \quad \frac{y_{n+s}}{e}=p^{\delta} H^{\prime \prime} \square .
$$

Then again $R_{e,\left(H^{\prime}, H^{\prime \prime}\right)}^{\prime \prime}$ is effectively bounded. Since this holds for each $\left(H^{\prime}, H^{\prime \prime}\right) \in \mathcal{H}$ the set $R_{e}$ is effectively bounded for each $e \mid y_{1} y_{2} \cdots y_{2 s}$. Hence $R$ is effectively bounded and this concludes the proof of the theorem.

4. A numerical example. We give an example where our method is applied with success to determine explicitly all solutions. To begin we prove a lemma.

(4.1) Lemma. Let $U_{n}, V_{n}$ be the Pell numbers for all $n \in \mathbb{Z}$. Then

(a) $\left\{n \neq 0 \mid U_{n}=3 \square\right\}=\{4\},\left\{n \mid V_{n}=3 \square\right\}=\emptyset$,

(b) $\left\{n \neq 0 \mid U_{n}=6 \square\right\}=\emptyset$.

Proof. (a) Let $U_{n}=3 \square$. By considering the sequence $U_{n}$ modulo 3 we see that 4 divides $n$. Let $n=4 h$, so $U_{n}=U_{2 h} V_{2 h}$, with $\operatorname{gcd}\left(U_{2 h}, V_{2 h}\right)=2$. Then either $V_{2 h}=2 \square$ or $U_{2 h}=2 \square$. So $h=1$ and $n=4$. If $V_{n}=3 \square$, since $2 \mid V_{n}$ but $4 \nmid V_{n}$ this is impossible.

(b) If $U_{n}=6 \square$ then $n=4 h$ and we have the following cases:

$$
\begin{array}{l|c|c|c}
U_{2 h}=3 \square & 6 \square & \square & 2 \square, \\
V_{2 h}=2 \square & \square & 6 \square & 3 \square .
\end{array}
$$

From (a) and the knowledge of $m$ such that $U_{m}=\square, 2 \square, V_{m}=\square, 2 \square$ we conclude that $n=0$.

(4.2) EXAMPLE. If $p$ is a prime, or $p=1$, if $x, y, z$ are positive integers and

$$
\left\{\begin{array}{l}
x^{2}-2 y^{2}=1 \\
x^{2}-p z^{2}=9
\end{array}\right.
$$

then $(x, y, z, p)=(99,70,24,17)$.

Proof. $\varepsilon=1+\sqrt{2}$ is the fundamental unit of $\mathbb{Z}[\sqrt{2}]$, let $P=2, Q=-1$, $U_{n}, V_{n}$ are the Pell numbers, $k_{2}=9$, so the method is applicable. $\varepsilon^{2}=$ $3+2 \sqrt{2}$ is the fundamental solution of the first equation, $x_{n}+y_{n} \sqrt{2}=$ $\varepsilon^{n+2}=V_{n+2} / 2+U_{n+2} \sqrt{2}$ and we work with $n$ even since $Q=-1$. We have:

$$
2 y_{n-2} y_{n+2}=p z^{2} \neq 0,
$$

that is, $U_{n} U_{n+4}=2 p \square \neq 0$.

(a) $p=2$, so $U_{n} U_{n+4}=\square$. If $n \equiv 2(\bmod 4)$ then $\operatorname{gcd}\left(U_{n}, U_{n+4}\right)=U_{2}$ $=2$, so $U_{n}=2 \square$ and $U_{n+4}=2 \square$, which is impossible. If $n \equiv 0(\bmod 4)$ then $\operatorname{gcd}\left(U_{n}, U_{n+4}\right)=U_{4}=12$, hence $U_{n}=3 \square, U_{n+4}=3 \square$, which is impossible by (4.1). 
(b) $p \neq 2$, so $U_{n} U_{n+4}=2 p \square$. If $n \equiv 2(\bmod 4)$ then $U_{n} \equiv U_{n+4} \equiv 2$ $(\bmod 4)$ so the 2 -adic value of $U_{n} U_{n+4}$ is even, hence $U_{n} U_{n+4}=2 p \square$ is impossible.

Now let $n \equiv 0(\bmod 4)$, so the following cases are possible:

$$
\begin{array}{c|c|c|c}
U_{n}=3 \square & 6 \square & 3 p \square & 6 p \square, \\
U_{n+4}=6 p \square & 3 p \square & 6 \square & 3 \square .
\end{array}
$$

(1) It was seen that $n=4$, hence $U_{8}=408=6 \times 17 \square$ so $p=17$, $x_{4}=V_{6} / 2=99, y_{4}=U_{6}=70$ and this gives the solution $(x, y, z, p)=$ $(99,70,24,17)$.

(4) $n+4=4, n=0$ which is impossible, since then $z=0$.

(2) and (3) are impossible as it was shown in Lemma (4.1).

As an exercise the reader may wish to show that if $x, y, z$ are positive, if $p$ is a prime number and if

$$
\left\{\begin{array}{l}
x^{2}-3 y^{2}=-3 \\
x^{2}-3 p z^{2}=-12
\end{array}\right.
$$

then $(x, y, z, p)=(3,2,1,7)$.

The reader may employ the same method to prove the original result of Ljunggren mentioned in the Introduction: if $x, y$ are positive integers, if $p$ is a prime number and $x^{4}-p y^{2}=1$ then $(x, y, p)=(3,4,5)$ or $(99,1820,29)$.

\section{References}

[1] J. H. E. Cohn, Squares in some recurrent sequences, Pacific J. Math. 41 (1972), 631-646.

[2] -, The Diophantine equation $x^{4}-D y^{2}=1, I I$, Acta Arith. 78 (1997), 401-403.

[3] W. Ljunggren, Einige Eigenschaften der Einheiten reeller quadratischer und reinbiquadratischer Zahlkörper mit Anwendung auf die Lösung einer Klasse unbestimmter Gleichungen vierten Grades, Det Norske Vid. Akad. Skr. I, 1936, no. 12 (1937), 73 pp. $(*)$

[4] -, Über die Gleichung $x^{4}-D y^{2}=1$, Arch. Math. og Naturvid. 45 (1942), no. 5, 61-70. $(*)$

[5] -, Zur Theorie der Gleichung $x^{2}+1=D y^{4}$, Det Norske Vid. Akad. Avh. I, 5 (1942), 27 pp. $(*)$

[6] -, Some remarks on the diophantine equations $x^{2}-D y^{4}=1$ and $x^{4}-D y^{2}=1$, J. London Math. Soc. 41 (1965), 42-44. (*)

[7] T. Nagell, Über die Darstellung ganzer Zahlen durch eine indefinite binäre quadratische Form, Arch. Math. 2 (1949/50), 161-165. (**)

(*) Reprinted in Collected Papers of Wilhelm Ljunggren, Paulo Ribenboim (ed.), Queen's Papers in Pure and Appl. Math. 115, Kingston, 2003.

(**) Reprinted in Collected Papers of Trygve Nagell, Paulo Ribenboim (ed.), Queen's Papers in Pure and Appl. Math. 121, Kingston, 2002. 
[8] T. Nagell, Bemerkung über die Diophanthische Gleichung $u^{2}-D v^{2}=c$, ibid. 3 (1952), 8-9. (**)

[9] A. Pethő, Perfect powers in second order linear recurrences, J. Number Theory 15 (1982), 5-13.

[10] P. Ribenboim, Pell numbers: squares and cubes, Publ. Math. Debrecen 54 (1999), 131-152.

[11] -, My Numbers, My Friends, Springer, New York, 2000.

[12] P. Samuel, Résultats élémentaires sur certaines équations diophantiennes, J. Théorie Nombres Bordeaux 14 (2002), 629-646.

[13] T. N. Shorey and C. L. Stewart, On the Diophantine equation $a x^{2 t}+b x^{t} y+c y^{2}=1$ and pure powers in recurrence sequences, Math. Scand. 52 (1983), 24-36.

[14] T. N. Shorey and R. Tijdeman, Exponential Diophantine Equations, Cambridge Univ. Press, Cambridge, 1986.

Department of Mathematics and Statistics

Queen's University

Kingston, Ontario, Canada

Received on 20.2.2001

and in revised form on 22.11.2004 